\title{
Elucidation of Distinct Roles of Guinea Pig CXCR1 and CXCR2 in Neutrophil Migration toward IL-8 and GRO $\alpha$ by Specific Antibodies
}

\author{
Kento Tanaka, ${ }^{*, a}$ Tomomi Yoshitomi, ${ }^{b}$ and Kazuki Hirahara ${ }^{a}$ \\ ${ }^{a}$ Biologics \& Immuno-Oncology Laboratories, Daiichi Sankyo Co., Ltd.; 1-2-58 Hiromachi, Shinagawa-ku, Tokyo \\ 140-8710, Japan: and ${ }^{b}$ End-Organ Disease Laboratories, Daiichi Sankyo Co., Ltd.; 1-2-58 Hiromachi, Shinagawa- \\ ku, Tokyo 140-8710, Japan.
}

Received November 26, 2016; accepted February 15, 2017

\begin{abstract}
Chemokine receptors CXCR1 and CXCR2 are conserved between guinea pigs and humans, but the distinct role of each receptor in chemotactic responses of neutrophils against chemokine ligands has not been elucidated due in part to the lack of specific inhibitors against these receptors in guinea pigs. In this study, we investigated the roles of guinea pig CXCR1 and CXCR2 on neutrophils in chemotactic responses to guinea pig interleukin (IL)-8 and growth-regulated oncogene (GRO) $\alpha$ by using specific inhibitory antibodies against these receptors. Neutrophil migration induced by IL-8 was partially inhibited by either antiCXCR1 antibody or anti-CXCR2 antibody. In addition, the migration was inhibited completely when both anti-CXCR1 and anti-CXCR2 antibodies were combined. On the other hand, neutrophil migration induced by GRO $\alpha$ was not inhibited by anti-CXCR1 antibody while inhibited profoundly by anti-CXCR2 antibody. These results indicated that CXCR1 and CXCR2 mediated migration induced by the IL-8 synergistically and only CXCR2 mediated migration induced by GRO $\alpha$ in guinea pig neutrophils. Our findings on ligand selectivity of CXCR1 and CXCR2 in guinea pigs are consistent with those in humans.
\end{abstract}

Key words guinea pig; CXCR1; CXCR2; neutrophil; antibody; chemokine

CXCR1 and CXCR2 are the chemokine receptors mainly expressed in leukocytes, such as neutrophils, and mediate migration and activation of the cells in response to the ligand stimulation. ${ }^{1)}$ The ligand selectivity of these receptors are known to be different, ${ }^{2)}$ for example, interleukin (IL)-8 and granulocyte chemotactic protein 2 (GCP-2) are common ligands of CXCR1 and CXCR2, and growth-regulated oncogene (GRO) $\alpha / \beta / \gamma(\mathrm{CXCL1} / 2 / 3)$, ENA-78 (CXCL5), and NAP-2 (CXCL7) are ligands selective for CXCR2. The expression levels of IL-8 or GRO $\alpha$ have been reported to increase at inflammation sites in respiratory diseases ${ }^{3,4)}$ and this suggested that leukocytes were recruited and activated by these ligands through both CXCR1 and CXCR2 or only CXCR2, depending on the ligand. In order to clarify the pathology of these diseases, the elucidation of the distinct functions of CXCR 1 and CXCR2 is important.

The guinea pig, which have often been utilized to understanding inflammatory diseases, ${ }^{5,6)}$ is considered to be an appropriate species for elucidating the functions of CXCR1 and CXCR2. Guinea pig CXCR1 (gpCXCR1) and CXCR2 (gpCXCR2) were previously cloned and their functions were confirmed by gpCXCR1- or gpCXCR2-transfected HEK293 cells. ${ }^{7,8)}$ Guinea pig IL-8 (gpIL-8) was cloned and reported to activate both gpCXCR1 and gpCXCR2, ${ }^{9)}$ in contrast to mice or rats, which do not have the ortholog of IL-8. Guinea pig $\operatorname{GRO} \alpha(\operatorname{gpGRO} \alpha)$ was also cloned, ${ }^{10)}$ and the selective activation of gpCXCR2 was confirmed by our group using gpCXCR1- or gpCXCR2-transfected Chinese hamster ovary (CHO)-K1 cells. ${ }^{11)}$ However, the analysis of the functions of gpCXCR1 and gpCXCR2 were conducted only in transfected cells, and the functions of gpCXCR1 and gpCXCR2 on the cells endogenously expressing these receptors, such as neutrophils, are not fully understood because the specific inhibitors against gpCXCR1 and gpCXCR2 were not found. ${ }^{12)}$
In this study we tried to clarify the distinct functions of the gpCXCR1 and gpCXCR2 in the migration of neutrophils by using specific antibodies that were generated and characterized in our previous study. ${ }^{11)}$ As a result, we demonstrated that the migration induced by gpIL- 8 was mediated by both gpCXCR1 and gpCXCR2, and the migration induced by gpGRO $\alpha$ was mediated by only gpCXCR2, which is consistent with the ligand selectivity of the receptors in humans.

\section{MATERIALS AND METHODS}

Production of Antibodies and Chemokines AntigpCXCR1 and gpCXCR2 monoclonal antibodies, AbCR1 (mouse immunoglobulin $\mathrm{G}$ (IgG)2a antibody) and AbCR2 (mouse IgG2b antibody) were generated by DNA immunization of mice using gpCXCR1 (NCBI Refseq: NM_001173416) and gpCXCR2 (NCBI Refseq: NM_001172875) expression vectors, pcDNA3.1/gpCXCR1 and pcDNA3.1/gpCXCR2, and a hybridoma technique using SP2 myeloma as described previously. ${ }^{11)}$ Mouse IgG2a isotype control antibody and mouse IgG2b isotype control antibody were purchased from Thermo Fisher Scientific (U.S.A.).

Guinea pig IL-8 and GRO $\alpha$ were produced by OrigamiB strain Escherichia coli using gpIL-8 (NCBI Refseq: NM_001173399) and gpGRO $\alpha$ (NCBI Refseq: NM_001172938) expression vectors pET11d/gpIL-8 and pET22b/gpGRO $\alpha$ as described previously. ${ }^{11)}$

Preparation of Guinea Pig Neutrophils Five- to sevenweeks-old female Hartley guinea pigs were used to obtain neutrophils. In brief, guinea pigs were euthanized by $\mathrm{CO}_{2}$ gas and lower limbs were dislocated. Skin and muscle were removed from femurs and tibias, and they were separated from the lower limbs. After rinsing with RPMI1640 supplemented with $100 \mathrm{U} / \mathrm{mL}$ penicillin, and $100 \mu \mathrm{g} / \mathrm{mL}$ streptomycin, the ends of 
bones were cut and bone marrow cells were recovered by flushing from both ends of the bone shafts with $10 \mathrm{~mL}$ RPMI1640 supplemented with $100 \mathrm{U} / \mathrm{mL}$ penicillin, and $100 \mu \mathrm{g} / \mathrm{mL}$ streptomycin using a 25 -gauge needle and $5 \mathrm{~mL}$ syringe.

Neutrophils were separated from bone marrow cells by density gradient centrifugation. In brief, $10 \mathrm{~mL}$ of Histopaque 1119 (Sigma-Aldrich, U.S.A.) was added to a $50 \mathrm{~mL}$ conical tube, and $10 \mathrm{~mL}$ of Histopaque 1077 (Sigma-Aldrich) and $25 \mathrm{~mL}$ of the recovered bone marrow cells were overlayed sequentially without disturbing the interfaces between each layer. The tube was centrifuged for $30 \mathrm{~min}$ at $2200 \mathrm{rpm}$ and $25^{\circ} \mathrm{C}$ without braking engaged. After the centrifugation, neutrophils were collected from the interface between Histopaque 1119 and Histopaque 1077 layers, and washed and resuspended with RPMI1640 supplemented 10\% fetal bovine serum (FBS) and $100 \mathrm{U} / \mathrm{mL}$ penicillin, and $100 \mu \mathrm{g} / \mathrm{mL}$ streptomycin. In the separated cells, the population of neutrophils was determined by the flow cytometry analysis to be 40 to $50 \%$.

All experimental procedures were performed in accordance with the in-house guidelines of the Institutional Animal Care and Use Committee of Daiichi Sankyo Co., Ltd. (Japan).

Flow Cytometry Analysis The expression of gpCXCR1 and gpCXCR2 on the neutrophils was assessed by flow cytometry analysis. Separated guinea pig neutrophils were resuspended in staining buffer, phosphate buffered saline (PBS) supplemented 5\% FBS. The antibodies were diluted with the staining buffer to the indicated concentration. The cells were incubated with the antibody solutions for $30 \mathrm{~min}$ at $4^{\circ} \mathrm{C}$, and washed with staining buffer. The cells were stained with $10 \mu \mathrm{g} / \mathrm{mL}$ of Alexa488-conjugated goat anti-mouse IgG antibody (Thermo Fisher Scientific) for $30 \mathrm{~min}$ at $4^{\circ} \mathrm{C}$. For the exclusion of dead cells, a LIVE/DEAD ${ }^{\circledR}$ Fixable Far Red Dead Cell Stain Kit (Thermo Fisher Scientific) was used. Fluorescence of the neutrophils was measured using a flow cytometer FC500 (Beckman Coulter, U.S.A.), and analyzed by FlowJo (FlowJo LLC, U.S.A.).

Migration Assay Separated guinea pig neutrophils were suspended in assay buffer, RPMI supplemented with $2 \% \mathrm{FBS}$, $100 \mathrm{U} / \mathrm{mL}$ penicillin, and $100 \mu \mathrm{g} / \mathrm{mL}$ streptomycin at a concentration of $2 \times 10^{7}$ cells $/ \mathrm{mL}$ and the indicated concentration of antibodies. GpIL- 8 and gpGRO $\alpha$ were diluted to the indicated concentrations with the assay buffer as a chemoattractant. A Transwell ${ }^{\circledR}$ with $5.0 \mu \mathrm{m}$ Pore Polycarbonate Membrane Insert was set onto the 24 -well plate, and $100 \mu \mathrm{L}$ of cell suspension was added to the insert followed by the addition of $600 \mu \mathrm{L}$ of chemoattractant to the lower well. The plate was incubated for $90 \mathrm{~min}$ at $37^{\circ} \mathrm{C}$ and $5 \% \mathrm{CO}_{2}$. After the incubation, the inserts were removed and $0.1 \%$ glutaraldehyde was added to the lower well for immobilization. Flow Count Fluorospheres (Beckman Coulter) were added to the lower well and the number of the migrated neutrophils was counted using an FC500 flow cytometer.

\section{RESULTS}

GPCXCR1 and GpCXCR2 Expression on the Guinea Pig Neutrophils In order to analyze the expression profile of gpCXCR1 and gpCXCR2 on the guinea pig neutrophils, we evaluated the binding of anti-gpCXCR1 antibody and anti-gpCXCR2 antibody, AbCR1 and AbCR2, respectively. Guinea pig neutrophils were incubated with each antibody and
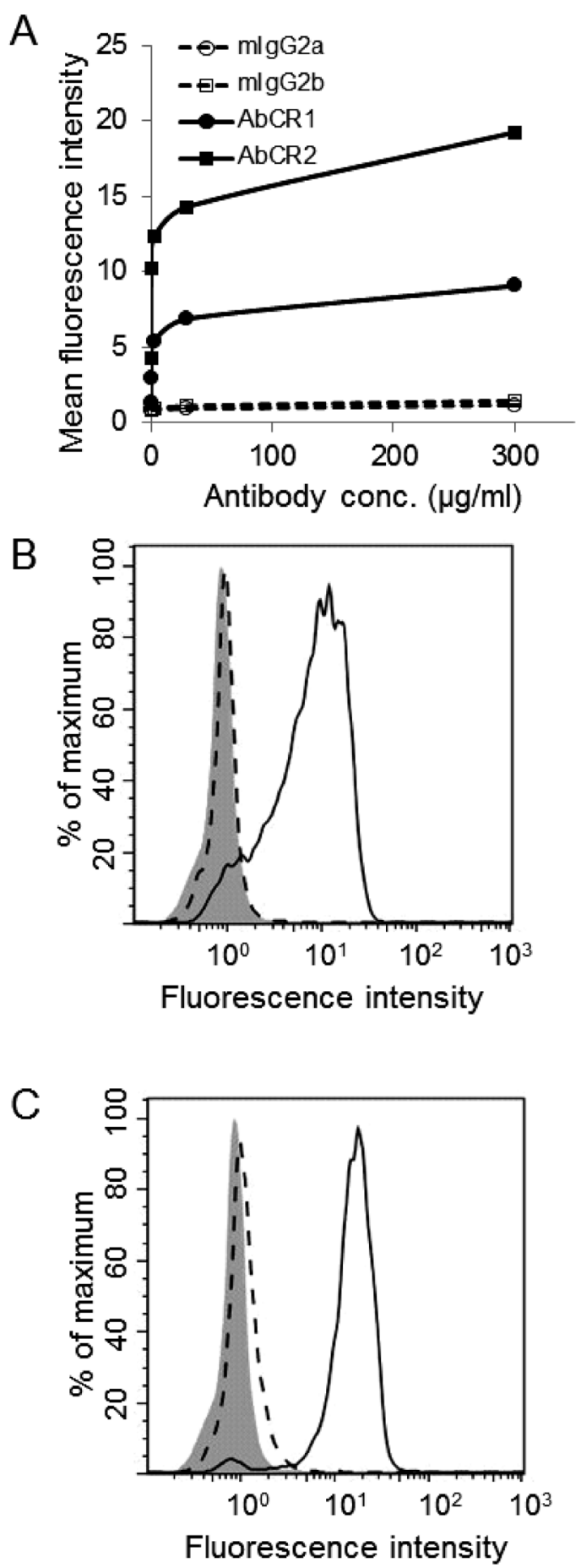

Fig. 1. Binding of Anti-gpCXCR1 and Anti-gpCXCR2 Antibodies to Guinea Pig Neutrophils

(A) Binding of anti-gpCXCR1 antibody, AbCR1 (filled circle with solid line), anti-gpCXCR2 antibodies, AbCR2 (filled square with solid line), mouse IgG2a isotype control (open circle with dashed line) and mouse IgG2b isotype control (open square with dashed line) at indicated concentrations to guinea pig neutrophils are presented with standard error of the mean (S.E.M.) ( $n=3)$. Binding of (B) $30 \mu \mathrm{g} / \mathrm{mL}$ of AbCR1 (solid line), mouse IgG2a isotype control (dashed line) and no antibody (grey filled) and (C) $30 \mu \mathrm{g} / \mathrm{mL}$ of AbCR2 (solid line), mouse IgG2b isotype control (dashed line) and no antibody (grey filled) at each $30 \mu \mathrm{g} / \mathrm{mL}$ are depicted as representative.

the bound antibodies were detected by flow cytometry. Both AbCR1 and AbCR2 bound to the neutrophils in a concentration-dependent manner, and the bindings of the antibodies saturated at $300 \mu \mathrm{g} / \mathrm{mL}$ (Fig. 1A). In addition, the histograms showed shifts in a single peak by AbCR1 (Fig. 1B) and 

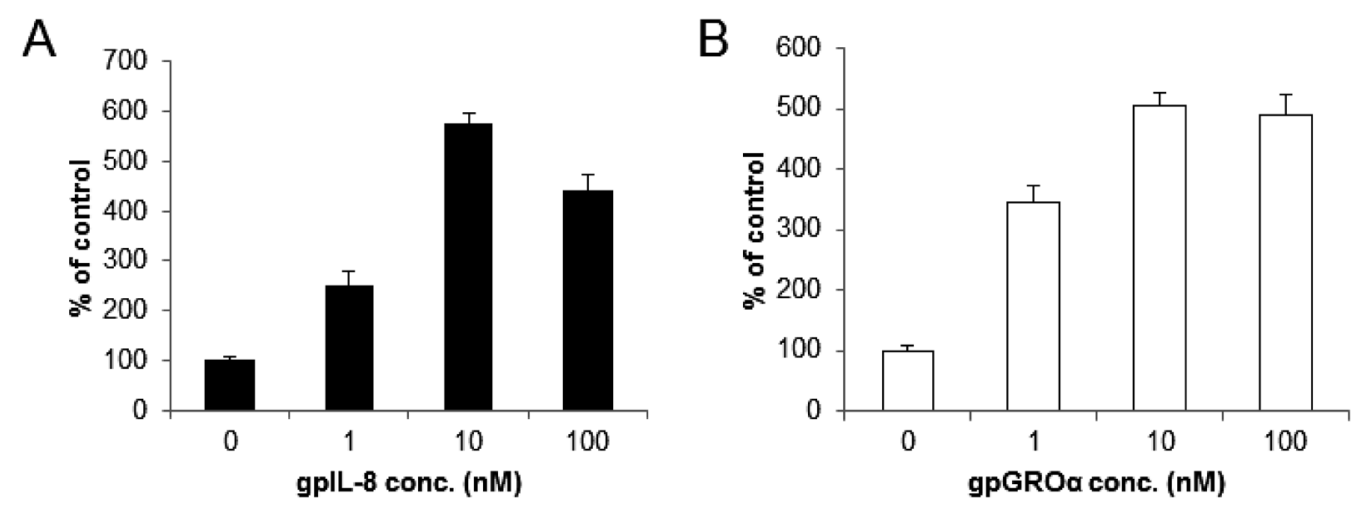

Fig. 2. Migration of Guinea Pig Neutrophils Induced by GpIL-8 and GpGRO $\alpha$

Migration of guinea pig neutrophils induced by (A) gpIL-8 (filled bar) and (B) gpGRO $\alpha$ (open bar) is presented. The cells were cultured for 90 min in a Transwell ${ }^{\circledR}$. The migrated neutrophils in the lower well were counted by flow cytometry. Spontaneous migrated cells without chemokine induction were set to $100 \%$ as control. The data are presented as the percent of migration to the control with S.E.M. $(n=3)$.
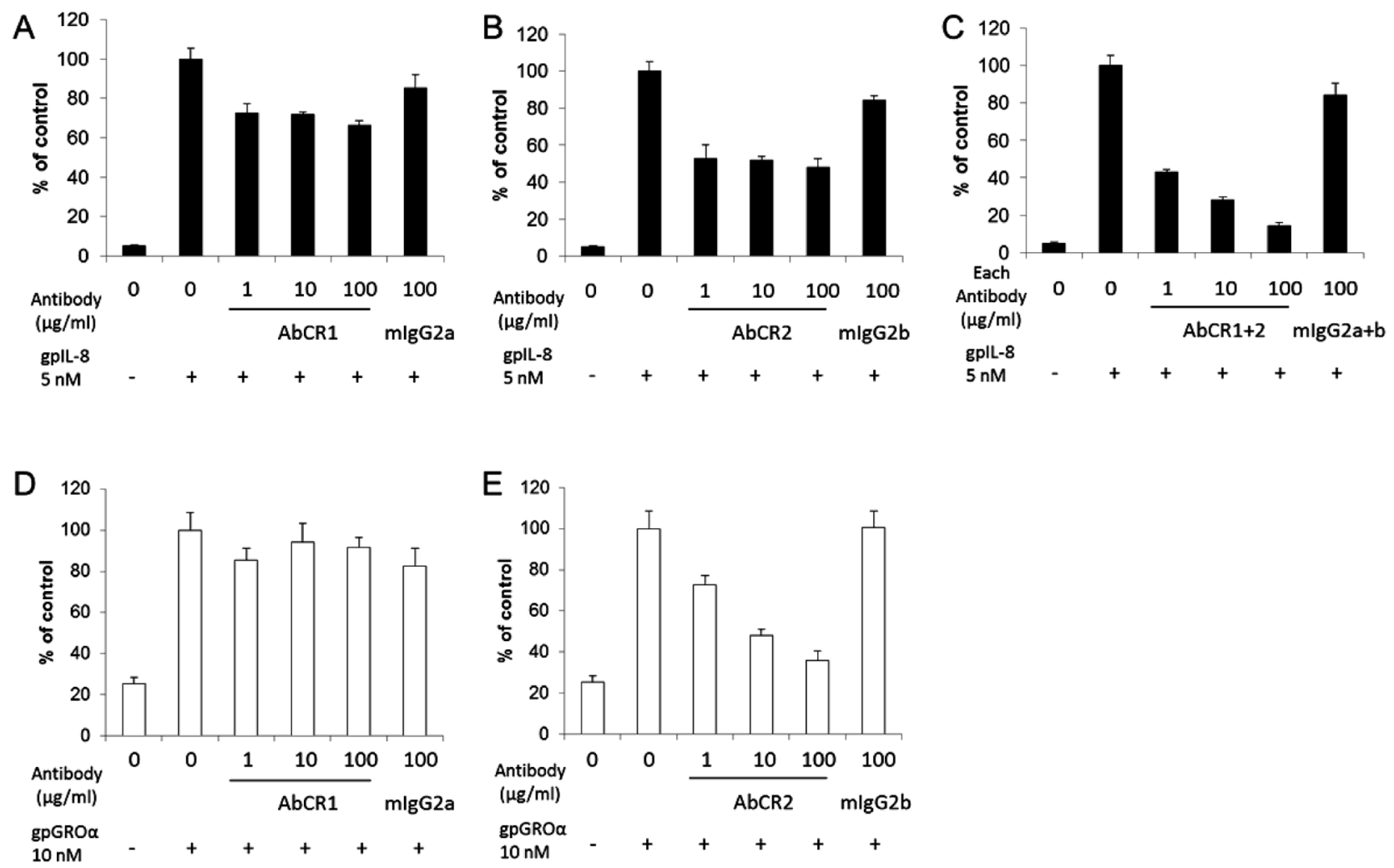

Fig. 3. Inhibitory Activity of Anti-gpCXCR1 and Anti-gpCXCR2 Antibodies against Migration of Guinea Pig Neutrophils

Migration of guinea pig neutrophils was induced by $5 \mathrm{~nm}$ of gpIL-8 (closed bar) in the presence of (A) AbCR1 or mouse IgG2a isotype control, (B) AbCR2 or mouse IgG2b isotype control, and $(\mathrm{C})$ combination of AbCR1 and AbCR2 or combination of mouse IgG2a and IgG2b isotype control at indicated concentrations. Migration of guinea pig neutrophils was induced by $10 \mathrm{~nm}$ of gpGRO $\alpha$ (open bar) in the presence of (D) AbCR1 or mouse IgG2a isotype control or (E) AbCR2 or mouse IgG2b isotype control at indicated concentrations. Chemokine-induced migrated cells in the absence of antibody were set to $100 \%$ as control. The data are presented as the percent of migration to the control with S.E.M. $(n=3)$.

AbCR2 (Fig. 1C) against neutrophils. These results indicated that the guinea pig neutrophils express homogeneously both gpCXCR1 and gpCXCR2 at the protein level.

Chemotactic Activity of GpIL-8 and GpGRO $\alpha$ against Guinea Pig Neutrophils We evaluated migration of the neutrophils toward gpIL-8 and gpGRO $\alpha$ in order to determine the chemotactic activity of these chemokines against the guinea pig neutrophils. For migration assay of the guinea pig neutrophils, a Transwell ${ }^{\circledR}$ system was used in which the neutrophils migrate toward lower wells filled with chemoattractant across the porous membrane insert. The number of migrated neutrophils in the lower well was counted by flow cytometry. As a result, both gpIL-8 and gpGRO $\alpha$ induced migration of guinea pig neutrophils in a concentration-dependent manner, and both migrations peaked at $10 \mathrm{~nm}$ of gpIL- 8 and gpGRO $\alpha$ (Figs. 2A, B).

Contribution of GpCXCR1 and GpCXCR2 on the Migration of the Guinea Pig Neutrophils We evaluated inhibitory activity of AbCR1 and AbCR2 on the migration of guinea pig neutrophils toward gpIL- 8 and gpGRO $\alpha$ in a migration assay using a Transwell ${ }^{\circledR}$ to elucidate the contributions of the gpCXCR1 and gpCXCR2. In the following assay, chemotactic responses of the neutrophils were induced by $5 \mathrm{nM}$ of gpIL- 8 or $10 \mathrm{~nm}$ of $\operatorname{gpGRO} \alpha$, which we then observed for sufficient 
migration to evaluate the inhibitory activity of the antibodies. Regarding the migration induced by gpIL-8, both AbCR1 and AbCR2 showed partial inhibition of neutrophil migration (Figs. 3A, B). Additionally, the combination of the AbCR1 and AbCR2 showed complete inhibition of the migration induced by gpIL-8 at each $100 \mu \mathrm{g} / \mathrm{mL}$ (Fig. 3C), which is sufficient concentration for occupation of the CXCR1 and CXCR2 on the neutrophils according to Fig. 1. On the other hand, the migration induced by $10 \mathrm{~nm}$ of $\operatorname{gpGRO} \alpha$ was not affected at all, even at a concentration of $100 \mu \mathrm{g} / \mathrm{mL}$ of AbCR1, but was inhibited completely at $100 \mu \mathrm{g} / \mathrm{mL}$ of AbCR2 (Figs. 3D, E). These results indicated that both gpCXCR1 and gpCXCR2 synergistically mediated the migration induced by gpIL- 8 and only gpCXCR 2 mediated the migration induced by gpGRO $\alpha$.

\section{DISCUSSION}

In this study, we clarified the roles of CXCR1 and CXCR2 in the migration of guinea pig neutrophils by specific antibodies. First, we demonstrated that the neutrophils expressed both CXCR1 and CXCR2 homogeneously, and IL-8 and GRO $\alpha$ induced migration of the neutrophils. The neutrophil migration induced by IL- 8 was inhibited partially by anti-CXCR1 and anti-CXCR 2 antibodies, and completely by the combination of these antibodies. This indicated that both receptors were involved in the chemotactic responses of neutrophils towards IL- 8 and that IL- 8 signals were mediated through only these two receptors, while the neutrophil migration induced by GRO $\alpha$ was inhibited by only the anti-CXCR 2 antibody.

This is the first demonstration that the distinct roles of CXCR1 and CXCR2 on the guinea pig neutrophils were elucidated by the specific inhibitory antibodies. As for small molecule inhibitors, Planagumà et al. reported that four selective inhibitors to human CXCR2 were found to inhibit gpCXCR1 and gpCXCR2 with a similar potency due to the interspecies difference. ${ }^{12)}$ It is known that there are anti-human CXCR1 and CXCR2 antibodies, however, no antibodies were found to cross-react with either guinea pig CXCR1 or CXCR2 due to the low homology between humans and guinea pigs. Therefore, there were no specific inhibitors against gpCXCR1 and gpCXCR2 other than AbCR1 and AbCR2, and these antibodies first showed the chemotactic functions of CXCR1 and CXCR2 distinctly.

Our finding that guinea pig neutrophil express CXCR1 and CXCR2 and respond to IL- 8 and GRO $\alpha$ reinforces the usefulness of guinea pigs being used as animal models for neutrophilic inflammatory diseases. In human neutrophil, CXCR1 and CXCR2 functions have been evaluated by anti-human CXCR1 and CXCR2 antibodies. Similar to our result, Jones et al. reported that calcium influx of the neutrophil by IL-8 was mediated through CXCR1 and CXCR $2,{ }^{13)}$ and Hammond et al. reported that migration of neutrophil by $\mathrm{GRO} \alpha$ was mediated through CXCR2 only. ${ }^{14)}$ In contrast, Hammond et al. reported that the migration of neutrophil by IL-8 was mediated mainly by CXCR1 and the contribution of the CXCR2 was weak. These results might be dependent on the IL- 8 assay concentration and affinity of the antibodies. Which receptor works preferentially against IL-8 in guinea pigs and humans remains to be elucidated through further study.

In conclusion, these results indicated that both CXCR 1 and CXCR2 function on the neutrophils of guinea pigs in response to their ligands similarly to how they do in humans, and this further suggested the increased value of the guinea pig as a neutrophilic inflammatory disease model.

Acknowledgments We would like to thank Ms. Ikue Iino and Mr. Yoshiyuki Kanari for their technical contribution. We would also like to thank Dr. Toshinori Agatsuma for his critical review of this manuscript.

Conflict of Interest Kento Tanaka, Tomomi Yoshitomi and Kazuki Hirahara are employees of Daiichi Sankyo Co., Ltd.

\section{REFERENCES}

1) Bizzarri C, Beccari AR, Bertini R, Cavicchia MR, Giorgini S, Allegretti M. ELR+CXC chemokines and their receptors (CXC chemokine receptor 1 and $\mathrm{CXC}$ chemokine receptor 2) as new therapeutic targets. Pharmacol. Ther., 112, 139-149 (2006).

2) Stillie R, Faroog SM, Gordon JR, Stadnyk AW. The functional significance behind expressing two IL-8 receptor types on PMN. $J$. Leukoc. Biol., 86, 529-543 (2009).

3) Yamamoto C, Yoneda T, Yoshikawa M, Fu A, Tokuyama T, Tsukaguchi K, Narita N. Airway inflammation in COPD assessed by sputum levels of interleukin-8. Chest, 112, 505-510 (1997).

4) Traves SL, Culpitt SV, Russell REK, Barnes PJ, Donnelly LE. Increased levels of the chemokines GROalpha and MCP-1 in sputum samples from patients with COPD. Thorax, 57, 590-595 (2002).

5) Zhao X, Town JR, Li F, Zhang X, Cockcroft DW, Gordon JR. ELR-CXC chemokine receptor antagonism targets inflammatory responses at multiple levels. J. Immunol., 182, 3213-3222 (2009).

6) Fricker M, Deane A, Hansbro PM. Animal models of chronic obstructive pulmonary disease. Expert Opin. Drug Discov., 9 629-645 (2014)

7) Catusse J, Faye P, Loillier B, Cremers B, Franck RM, Luccarini JM, Pruneau D, Paquet JL. Cloning and characterization of guinea pig interleukin-8 receptor. Biochem. Pharmacol., 66, 1171-1180 (2003).

8) Takahashi M, Jeevan A, Sawant K, McMurray DN, Yoshimura T. Cloning and characterization of guinea pig CXCR1. Mol. Immunol., 44, 878-888 (2007).

9) Yoshimura T, Johnson DG. cDNA cloning and expression of guinea pig neutrophil attractant protein-1 (NAP-1). NAP-1 is highly conserved in guinea pig. J. Immunol., 151, 6225-6236 (1993).

10) Yoshimura T, Takeya M, Ogata H, Yamashiro S, Modi WS, Gillitzer R. Molecular cloning of the guinea pig GRO gene and its rapid expression in the tissues of lipopolysaccharide-injected guinea pigs. Int. Arch. Allergy Immunol., 119, 101-111 (1999).

11) Tanaka K, Yoshimura C, Shiina T, Terauchi T, Yoshitomi T, Hirahara K. Generation and characterization of specific antibodies against guinea pig CXCR1 or CXCR2. Monoclon. Antib. Immunodiagn. Immunother., in press

12) Planagumà $\mathrm{A}$, Domènech $\mathrm{T}$, Pont $\mathrm{M}$, Calama $\mathrm{E}$, García-González V, López R, Aulí M, López M, Fonquerna S, Ramos I, de Alba J, Nueda A, Prats N, Segarra V, Miralpeix M, Lehner MD. Combined anti CXC receptors 1 and 2 therapy is a promising anti-inflammatory treatment for respiratory diseases by reducing neutrophil migration and activation. Pulm. Pharmacol. Ther., 34, 37-45 (2015).

13) Jones SA, Wolf M, Qin S, Mackay CR, Baggiolini M. Different functions for the interleukin 8 receptors (IL-8R) of human neutrophil leukocytes: NADPH oxidase and phospholipase D are activated through IL-8R1 but not IL-8R2. Proc. Natl. Acad. Sci. U.S.A., 93, 6682-6686 (1996).

14) Hammond ME, Lapointe GR, Feucht PH, Hilt S, Gallegos CA, Gordon CA, Giedlin MA, Mullenbach G, Tekamp-Olson P. IL-8 induces neutrophil chemotaxis predominantly via type I IL-8 receptors. J. Immunol., 155, 1428-1433 (1995). 